\title{
Les outills mathématiques de la gestion des systèmes aquifères
}

\author{
par Pierre Combes, Patrick Goblet, Emmanuel Ledoux
}

Centre d'Informatique Géologique, Ecole des Mines de Paris, Fontainebleau

\section{INTRODUCTION}

La modélisation des flux dans la géosphère intéresse de nombreux problèmes pratiques en sciences de l'eau qui se posent de manière quantitative à l'ingénieur. Parmi ceux-ci, nous citerons: l'évaluation et la gestion des ressources en eau, la connaissance des pollutions accidentelles ou chroniques des systèmes aquiferes, l'analyse de la sûreté des stockages de déchets notamment toxiques ou radioactifs. l'étude d'impact de stockages d'hydrocarbures liquides ou gazeux, l'étude du comportement hydraulique et thermique des systèmes d'exploitation géothermique.

Afin d'apporter des réponses quantitatives aux problèmes posés, des outils de simulation numérique ont été développés et ont atteint actuellement des degrés de sophistication élevés, permettant de prendre en compte une grande variété de processus et de structures inhérentes à la complexité du milieu naturel.

Nous évoquerons dans la suite de cet article, en nous appuyant sur des exemples, le principe des outils de simulation les plus répandus et la portée pratique des phénomènes qu'ils permettent d'étudier dans le cadre des systèmes aquifères profonds.

\section{II 圈 LES SPÉCIFICITÉS DES NAPPES PROFONDES}

En milieu sédimentaire, les conditions de gisement des nappes profondes sont déterminées par la structure géologique des bassins qui peuvent être considérés à grande échelle comme une alternance de couches perméables et de couches

The mathenatical instruments for the modelling of aquiferous networks which have been developped for more than wenty years according to the functioning and operating constraints of such swstems.

The former instruments for the simulation which were inspired by modelling techics for oil tanks, have first studied the problems related to the flow and enabled researchers to realise many applications in the field of water supply.

The necessity to handle water quality problems which are bound with natural genchimical characteristics of the aquifers or with the luman influence (geological storage for instance) has generated the creation of more sophisticated nodels, connecting the flow with the transport of elements in solution which can interacte with the nilicu.

At the present time, a great number of tools for the simulation are proposed by research institutions and are nore and more being used by research deparments to solve successfully the practical problems laid down by deep waterbeaning-bed management in quantity and quality.

By using examples, this communication tries to show the diversity of the existing nodels and their applica tion scope by privileging the conceptual aspect of the instruments in comparison with the digital aspect. 


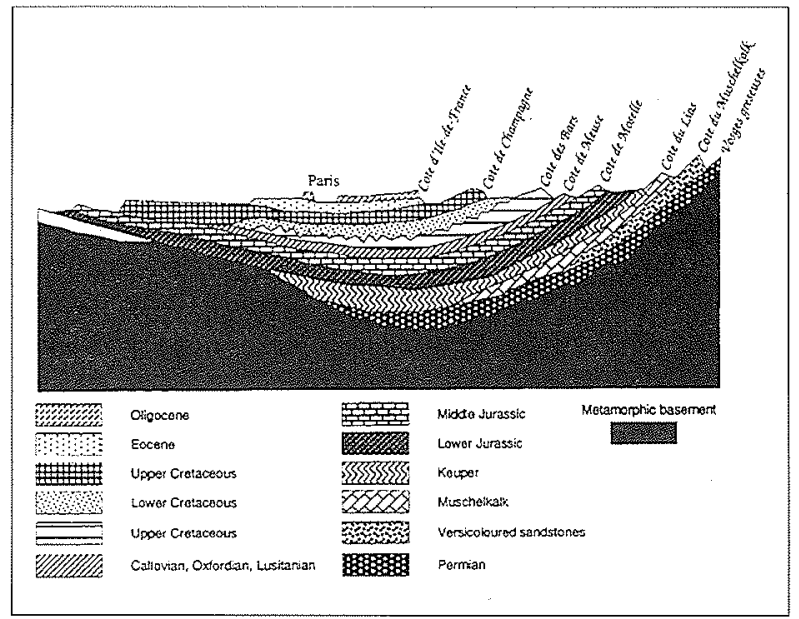

1. Structure géologique multicouche du Bassin parisien.

moins perméables ou même très peu perméables. Une coupe simplifiée du Bassin parisien (fig. I) permet ainsi de mettre en évidence des structures empilées relativement continues parmi lesquelles se distinguent de grands horizons perméables tels que les aquifères du Trias, du Dogger, du Crétacé moyen (Albien) ou du Crétacé supérieur (Craie). Entre ces horizons aquifères, se développent des couches dites semiperméables à dominante argileuse qui isolent hydrauliquement d'une manière plus ou moins efficace les aquifères les uns par rapport aux autres. Ainsi, dans le Bassin parisien, le Lias Supérieur et le Callovo-Oxfordien forment des structures marneuses très peu perméables et très continues.

Compte tenu de leurs conditions de gisement, nous résumerons comme suit les spécificités hydrogéologiques essentielles des nappes profondes:

- L'alimentation des couches aquifères s'effectue d'une manière générale sur les zones d'affleurement des horizons perméables en bordure des bassins sédimentaires. Ce mécanisme peut être actuel lorsque les conditions climatologiques s'y prêtent comme dans le Bassin parisien. Il peut être ancien, et se trouver ainsi négligeable à l'heure actuelle, dans les régions arides ou semi-arides. Dans ce demier cas, la ressource en eau s'apparente à une ressource minière, son renouvellement étant faible ou nul.

- Comme le montrent les cartes piézométriques à grande échelle, l'écoulement général des nappes profondes est conditionné par la drainance verticale à travers les couches semi-perméables. Cette situation confère à l'écoulement à l'échelle du bassin une structure tridimensionnelle alors que ce même écoulement peut être décrit avec une bonne approximation à deux dimensions au sein des aquiferes (Emsellem, 1967) dans la mesure où il se trouve canalisé par les couches semi-perméables environnantes.

- En fonction des distances entrant en ligne de compte, les temps de séjour des eaux au sein des aquifères profonds peuvent être très importants. Des mesures de ${ }^{14} \mathrm{C}$ réalisées dans des forages géothermiques de la nappe du Dogger en région parisienne montrent des âges apparents de l'eau de plusieurs dizaines de milliers d'années (Wei et al., 1990) tandis qu'un gradient observable d'est en ouest de ce même traceur atteste la présence d'un écoulement au sein de cet aquifère.

- L'ancienneté des eaux et la lenteur des écoulements favorisent l'équilibrage de la composition chimique de l'eau avec la roche encaissante. Il en résulte que plus les nappes sont profondes, plus leur minéralisation est susceptible d'être accentuée, ce qui peut conduire à rendre les eaux impropres à l'utilisation. Ce phénomène se trouve accru par des contacts possibles avec les formations salifères, comme cela est mis en évidence pour la nappe du Dogger dans le Bassin de Paris (Wei et al, 1990). Ces problèmes de qualité de l'eau pouvant évoluer à plus ou moins long terme lorsque l'hydrodynamisme est modifié par l'exploitation, doivent être pris en compte dans l'étude de la ressource en eau des nappes profondes.

- Le schéma général d'écoulement du bassin peut être localement perturbé essentiellement sous l'effet de variations de la densité de l'eau. Trois causes essentielles sont responsables de la variation de densité : la pression, la température et la concentration en sels dissous. L'effet de la salinité est par exemple bien visible dans l'aquifère du Dogger en région parisienne où la concentration varie rapidement entre 5 et $20 \mathrm{~g} / 1$ sous l'effet d'arrivées d'eau par drainance en provenance du Trias. La carte piézométrique à l'échelle du champ géothermique s'en trouve notablement perturbée et I'on peut constater des gradients hydrauliques locaux en sens inverse du gradient régional à l'échelle du bassin.

- Le schéma d'écoulement à l'échelle du bassin est également influencé par l'hydrodynamisme très particulier des horizons semi-perméables. En effet, ceux-ci possèdent une diffusivité très faible (rapport entre la perméabilité et le coefficient d'emmagasinement spécifique) qui leur confère un temps de réponse hydraulique très lent. Dans ces conditions. la pression de l'eau au sein d'un milieu très peu perméable peut être le reflet d'événements hydrogéologiques très anciens survenus par exemple au cours de la constitution du bassin ou au cours d'épisodes climatiques impliquant des conditions d'alimentation du système aquifère très différentes de l'actuel. Ce phénomène qualifié de «pressions anormales » est bien connu des pétroliers qui sont amenés à investiguer des milieux peu perméables, mais beaucoup moins connu des hydrogéologues qui par définition explorent les aquiferes dont le temps de réponse hydraulique est beaucoup plus rapide. Nous retiendrons de cette observation que selon les échelles de temps et d'espace avec lesquelles on étudie un système aquifère profond, il peut être nécessaire de considérer des mécanismes paléohydrogéologiques.

En résumé de ce qui précède, il apparaît que les mécanismes mis en jeu dans les systèmes aquifères profonds sont nombreux et complexes. Selon les exigences du problème posé, les modèles utilisés devront donc prendre en compte des mécanismes couplés à des degrés divers. Nous allons à présent illustrer ces propos par des exemples d'études de nappes profondes.

\section{PRINCIPES GÉNÉRAUX DE LA MODÉLISATION DES AQUIFÈRES PROFONDS : MULTICOUCHE}

Ces modèles s'adressent aux structures hydrogéologiques à grande échelle, et plus particulièrement au cas des bassins sédimentaires comportant plusieurs aquifères superposés. L'échelle du modèle peut varier dans d'assez larges proportions, disons pour fixer les idées, de quelques $\mathrm{km}^{2}$ à quelques centaines de milliers de $\mathrm{km}^{2}$ pour un grand bassin sédimentaire.

Dans de tels systèmes la conceptualisation des écoulements fait généralement appel à la notion de structure multicouche. L'alternance de couches sédimentaires de lithologie variée amène à définir des aquifères où s'effectue l'essentiel des circulations souterraines subhorizontales, et des semiperméables séparant les aquifères où les circulations horizontales sont négligeables, mais qui assurent les transferts de l'eau entre aquifères, à l'échelle régionale. 
L'idéalisation d'une telle structure aboutit au modèle multicouche constitué par un empilement de couches subhorizontales où l'écoulement est bidimensionnel (écoulement en nappe), reliées entre elles par des écoulements subverticaux monodimensionnels. Remarquons que la schématisation multicouche ne constitue qu'une simplification, en vue d'un allègement des calculs ultérieurs, d'une structure tridimensionnelle en mettant à profit les contrastes de perméabilité souvent rencontrés dans la nature. Lorsque ces contrastes n'existent pas, ou bien lorsqu'il s'avère nécessaire de décrire le comportement hydrodynamique d'un aquifère dans son épaisseur, on peut avoir recours à une modélisation tridimensionnelle complète, ce qui en fait, revient à accroître le nombre de couches.

La figure 2 illustre cette schématisation, sur l'exemple de la modélisation des aquifères tertiaires et jurassiques du Bassin aquitain. Dans cet exemple, huit couches aquifères ont été retenues pour couvrir une superficie cumulée de $250000 \mathrm{~km}^{2}$ (Besbès, 1976).

Une bonne identification de la structure conditionne la représentativité du modèle. Le travail du modélisateur doit donc débuter par une étude hydrogéologique approfondie dont la conclusion amènera à proposer une ou parfois plusieurs interprétations qui seront traduites à travers le modèle. Il convient cependant de noter que le choix de la bonne structure dépend du problème posé.

Les modèles de ressources en eau peuvent exiger la prise en compte d'un vaste domaine. Cela est d'autant plus vrai que le modèle intéressera des aquifères plus profonds, dont les conditions aux limites physiques se situeront parfois à grande distance. Il n'est pas rare, dans ces conditions de traiter des superficies de plusieurs dizaines de milliers de $\mathrm{km}^{2}$.

Les conséquences de l'ampleur d'un modèle sont de deux ordres:

- En premier lieu, le problème posé peut ne concerner qu'une petite partie du domaine d'étude. Il en résultera vraisemblablement une hétérogénéité dans la connaissance des caractéristiques du système se traduisant par une concentration des données sur quelques secteurs particuliers. La représentativité du modèle calé à l'aide d'une information hétérogène risque d'être compromise dans certains secteurs.
- En second lieu, les techniques de calcul qui sont employées ne diffèrent pas selon l'étendue du domaine modélisé. La mise en auvre de ces techniques, fondées sur la discrétisation spatiale, conduira ainsi à adopter un ordre de grandeur identique pour le nombre des éléments discrets ou mailles quel que soit le type de problème envisagé ; ce nombre étant en fait déterminé plus par la capacité de traitement des ordinateurs et la quantité de données à saisir que par la question posée.

Compte tenu de ce qui précède, une méthode de discréti sation particulièrement efficace consiste à découper les couches aquiferes en mailles carrées emboîtées en adaptant la géométrie du maillage aux spécificités de l'hétérogénéité du problème (fig. 3).

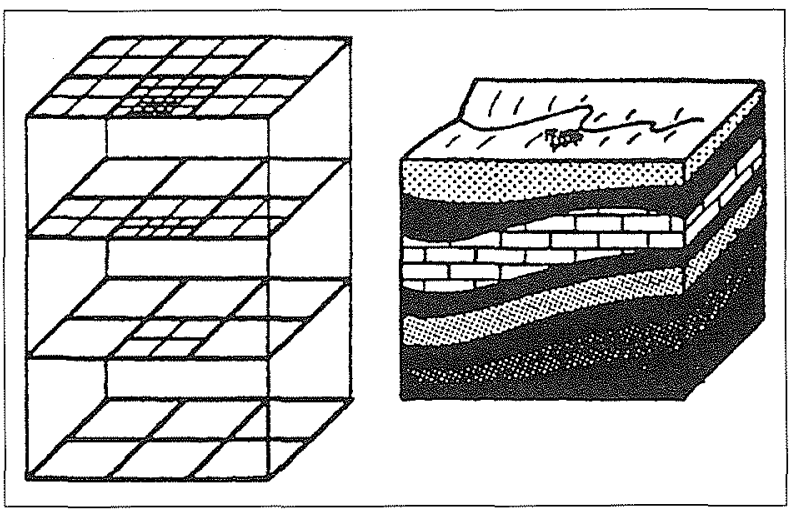

3. Exemple de discrétisation d'une structure multicouche en mailles carrées emboîtées.

La résolution des équations d'écoulement avec drainance peut ensuite être, par exemple, effectuée par la méthode des différences finies (Marsily et al., 1978 ; Ledoux, 1986).

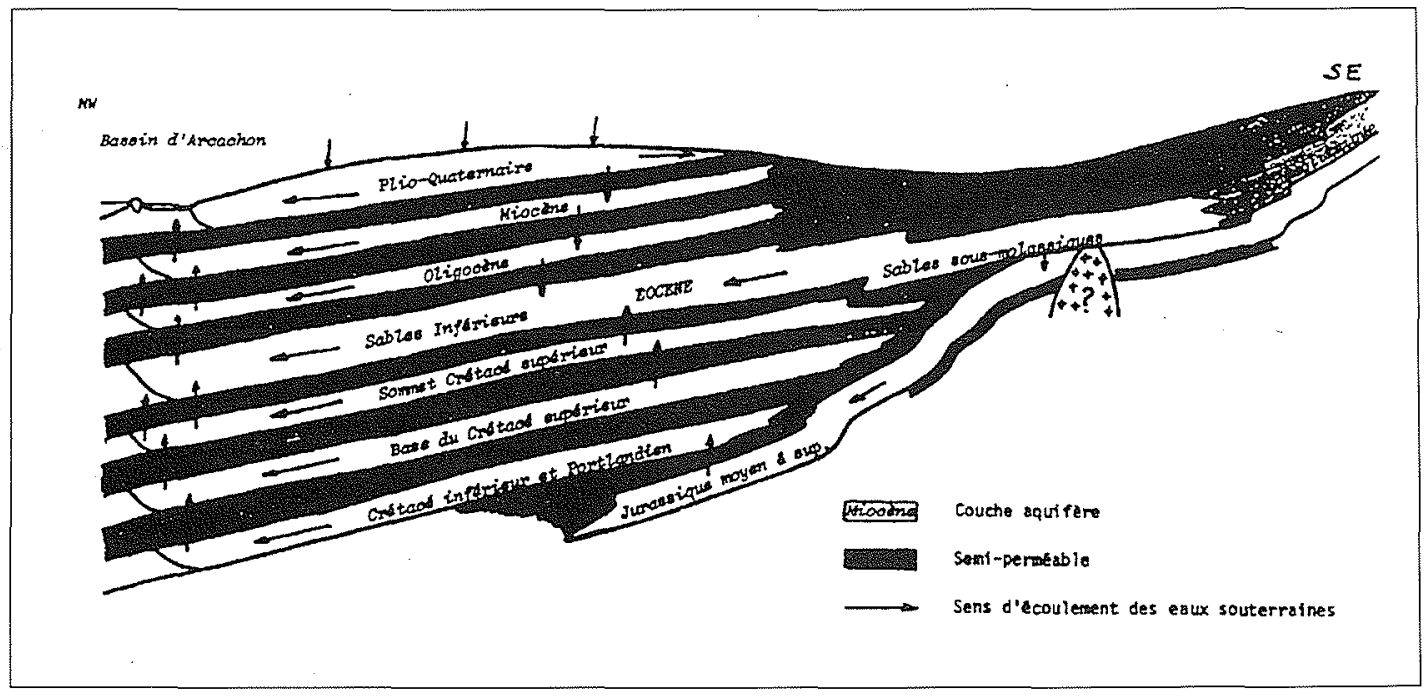

2. Schéma de la structure multicouche des aquifères tertiaires, crétacés et jurassiques du Bassin aquitain (d'après Besbès, 1976). 


\section{LA VALIDATION DES MODĖLES MULTICOUCHES}

Dans le cas des aquifères profonds, la validation du modèle pose un problème crucial. En effet, le bilan en eau du système hydrogéologique ne peut être contraint par aucune mesure directe. Le débit d'alimentation n'est pas mesurable car la quantité d'eau pénétrant dans les couches profondes n'est qu'une fraction, en général petite, de la pluie efficace disponible à l'affleurement. Le débit aux exutoires est également inobservable car il se mélange avec les eaux des aquifères superficiels. La seule possibilité consiste donc à caler le modèle sur des données piézométriques dans l'espace et dans le temps en tenant compte des mesures disponibles pour les paramètres hydrodynamiques.

Dans la schématisation multicouche, ces paramètres sont la transmissivité et le coefficient d'emmagasinement des aquifères ainsi que la diffusivité des semi-perméables. Cette dernière grandeur est en pratique inaccessible par des essais in situ, à l'échelle où il conviendrait d'en disposer et les mesures de transmissivité et de coefficient d'emmagasinement en aquifère sont rares si l'on considère la densité des forages profonds qu'il est possible de réaliser. On conçoit donc la difficulté de valider un modèle représentatif d'un aquifere profond.

Une méthode consiste à caler le modèle en régime transitoire sur des historiques piézométriques de longue durée résultant de perturbations naturelles ou anthropiques connues, mais ceci suppose des périodes d'observation étendues rarement disponibles. De surcroît, plus l'historique est long, plus intervient te rôle hydraulique transitoire des semi-perméables présentant des constantes de temps élevées, et plus la représentation de ces semi-perméables doit être soignée.

Une autre méthode consiste à utiliser les informations fournies par l'étude de traceurs naturels de l'écoulement. Cette approche est illustrée sur l'exemple du Bassin parisien (Wei, 1990). Un modèle maillé en différences finies comprenant 6 couches est construit pour représenter les aquifères du Trias, du Dogger du Lusitanien, du Portlandien, du Néocomien et de l'Albien (fig. 4). Un premier ajustement du modèle est obtenu en calant la piézométrie de la nappe du Dogger, assez bien connue grâce aux forages pétroliers et aux exploitations géothermiques, et en simulant les effets du rabattement de près de $120 \mathrm{~m}$ induit dans la nappe de l'Albien consécutivement à une exploitation de 140 années de cette nappe en région parisienne. Les vitesses d'écoulement calculées à l'aide du modèle sont ensuite utilisées pour simuler le transport convectif de trois traceurs naturels ayant des origines variées dans la nappe du Dogger : le sel provenant par drainance ascendante de l'aquifère du Trias, l'Hélium issu de la radioactivité naturelle d'émetteurs contenus dans les sédiments ( $U$ et $\mathrm{Th}$ ) enfin le ${ }^{1+} \mathrm{C}$ radiogénique introduit par les zones d'alimentation des nappes.

La bonne adéquation entre les concentrations mesurées en ces traceurs et les valeurs calculées par le modèle valide le bilan en eau dans la nappe du Dogger et par voie de conséquence la distribution à grande échelle du champ de transmissivité des aquiferes et de perméabilité des semiperméables (fig. 5).

Cette méthode semble prometteuse en particulier pour l'étude des risques inhérents aux stockages profonds pour lesquels les vitesses d'écoulement doivent être correctement évaluées.

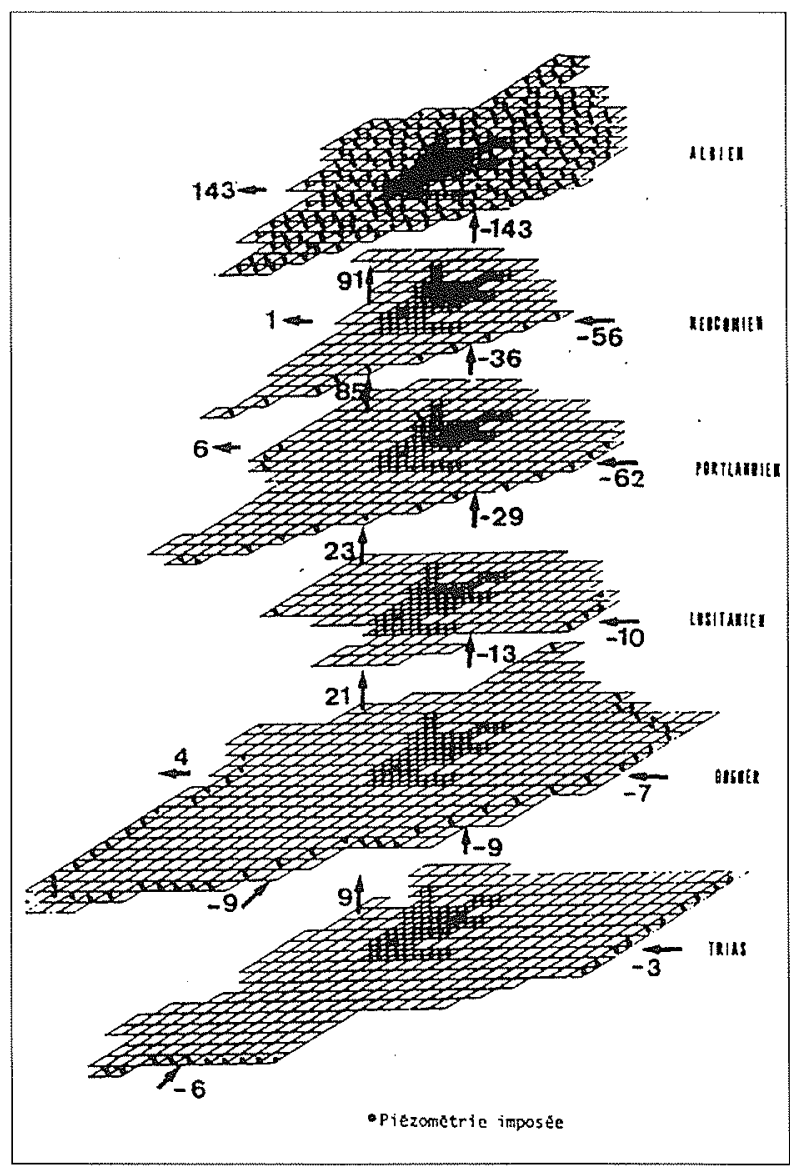

4. Structure du modèle multicouche du Bassin parisien et bilan des flux $(1 / s)$ en régime permanent (d'après Wei, 1990).

\section{$V$ UN EXEMPLE DE MÉCANISMES COUPLÉS : L'ÉVOLUTION À LONG TERME DE LA QUALITÉ DES EAUX SOUTERRAINES}

La plaine alluviale de Kairouan, en Tunisie, s'étend sur près de $3000 \mathrm{~km}^{2}$. Elle est constituée par une cuvette d'effondrements comblée par des dépôts détritiques continentaux d'âge plio-quaternaire. La sédimentation y est lenticulaire et formée d'alternances de sables plus ou moins grossiers et de marnes sur une épaisseur de $500 \mathrm{~m}$. On distingue un réservoir profond capté par forages et un niveau aquifère phréatique. Ces nappes sont essentiellement alimentées par les crues de deux oueds tandis que la reprise par évaporation constitue l'unique exutoire naturel, principalement au nord et au nord-est du domaine (Besbès et al., 1976).

En amont, la nappe profonde est alimentée par le niveau supérieur ; par contre, en aval, l'aquifëre profond se met en charge et percole dans la nappe phréatique.

La mise en valeur des terres agricoles entraînera dans l'avenir une surexploitation de la nappe profonde. Les rabattements résultant des pompages risqueront alors d'engendrer une contamination des eaux profondes par les eaux plus salées. de la nappe phréatique lorsque les gradients de niveaux piézométriques s'inverseront à l'aval.

Pour quantifier ce risque, une simulation de l'effet de ce régime de surexploitation a été réalisée au moyen d'un 

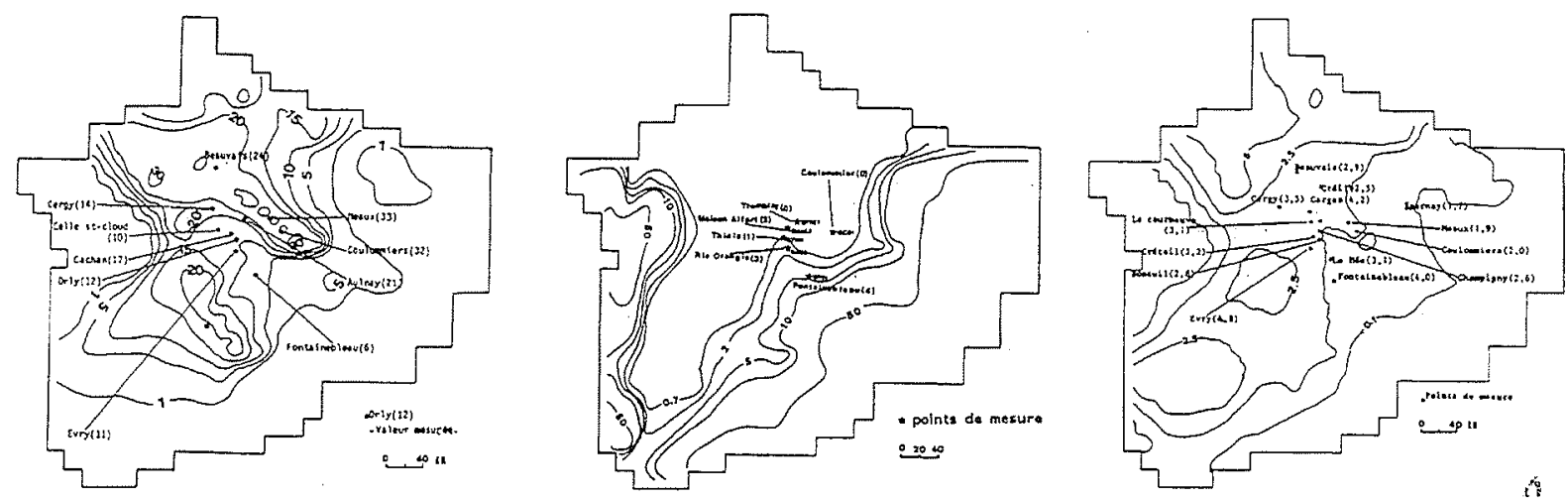

5. Simulation du transport de sel, d'hélium et de ${ }^{14} \mathrm{C}$ dans la nappe du Dogger. Comparaison entre valeurs calculées et observées (d'après Wei, 1990).

modèle maillé décrivant les transferts de matière dissoute à l'échelle régionale.

Le modèle considère une restriction aux simples échanges par convection du mouvement d'un corps dissous selon la théorie de la dispersion.

Les phénomènes sont schématisés en se limitant à la description du mouvement des sels dans la nappe profonde en supposant la concentration de la nappe phréatique invariante dans le temps. La concentration de l'eau pompée dans les forages provient du mélange qui s'effectue entre l'eau arrivant par drainance de la nappe phréatique et l'eau emmagasinée dans la porosité de la nappe profonde. Une épaisseur de $50 \mathrm{~m}$ pour une porosité de $5 \%$ ayant été attribuée à l'aquifère capté, les résultats des simulations recherchés sur 20 ans montrent un effet peu marqué des exploitations, l'augmentation maximale de concentration restant de l'ordre de $500 \mathrm{mg} / \mathrm{l}$ (fig.6).

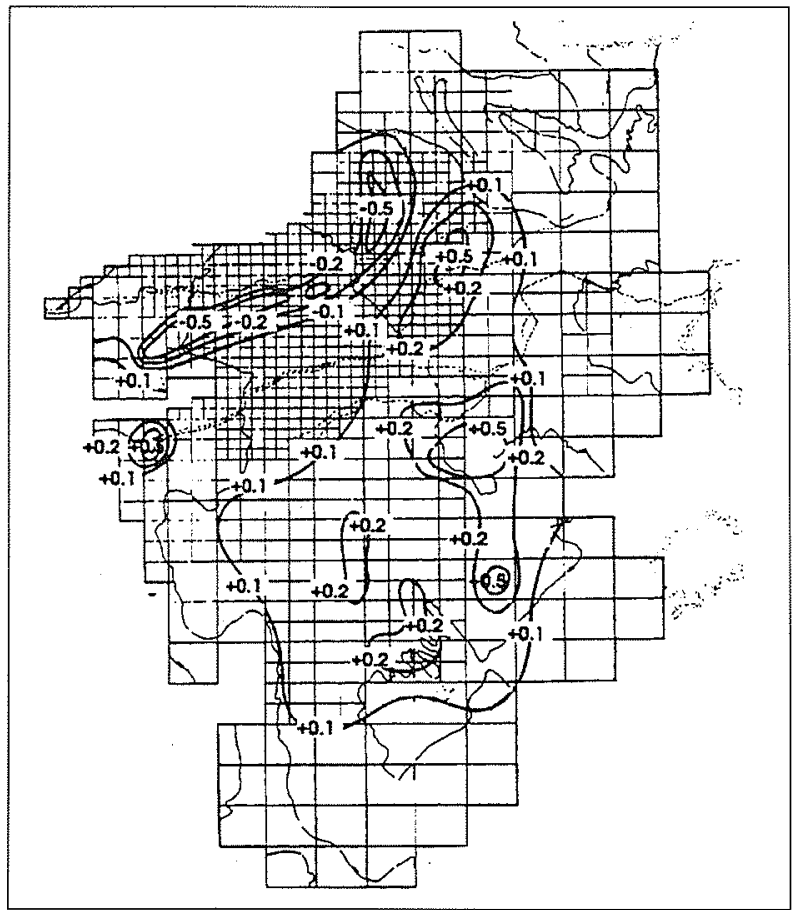

6. Variation calculée de la salinité de la nappe profonde (exprimée en $\mathrm{g} / \mathrm{l}$ ) de la plaine de Kairouan (Tunisie) après 20 ans d'exploitation (d'après Besbès, 1976).
Cette approche très globale néglige plusieurs phénomènes reconnus comme influençant le transfert des matières en solution dans un milieu poreux. En effet, aucune dispersion, ni aucun mécanisme d'interaction entre les sels dissous et la roche n'ont été considérés étant donné la connaissance expérimentale du système. Un tel modèle, bien que basé sur une approche conceptuelle, ne doit donc être utilisé que comme un indicateur des zones à risque dans le cadre d'une modélisation régionale à l'échelle d'un bassin hydrogéologique.

Une simulation plus fine prenant en compte les mécanismes de convection et de diffusion-dispersion est nécessaire pour étudier par exemple le transport du méthane en solution en provenance d'un stockage souterrain de gaz naturel. Les cycles d'exploitation du stockage constituent une réserve de gaz résiduel dans la nappe qui est progressivement entraînée par dissolution sous l'effet de l'écoulement naturel. Compte tenu des faibles vitesses d'écoulement, ce phénomène est susceptible de perdurer pendant un long laps de temps après l'arrêt de l'exploitation. La figure 7 montre un exemple de calcul de la concentration en méthane basé sur la résolution par la méthode des éléments finis de l'équation de la dispersion en 2 dimensions selon une coupe verticale passant par l'emprise du stockage.

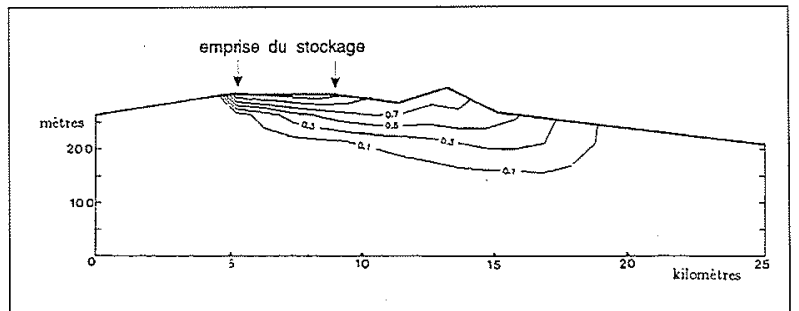

7. Simulation du panache de gaz dissous par lessivage du gaz résiduel d'un stockage en aquifère (concentration exprimée en $\mathrm{Sm}^{3} / \mathrm{m}^{3}$.

\section{VI 罝 UN EXEMPLE DE MÉCANISMES COUPLÉS : LA COMPACTION D'UN AQUIFÈRE MULTICOUCHE}

L'alimentation en eau de la ville de Mexico est assurée depuis les années 1930 par l'exploitation d'un aquifère fluviatile profond surmonté par des argiles lacustres dépassant $200 \mathrm{~m}$ d'épaisseur. Les rabattements importants de plusieurs 
dizaines de mètres engendrés dans l'aquifère dans un laps de temps relativement court ont causé l'apparition de « pressions anormales » dans le semi-perméable sus-jacent impliquant une compaction progressive des argiles. Cette compaction se traduit actuellement par une subsidence de près de $10 \mathrm{~m}$ en plein centre ville de Mexico. Dans le but de simuler l'évolution à long terme de la ressource en eau et ses conséquences sur la stabilité mécanique, un modèle multicouche représentant l'aquifère profond et le semi-perméable, totalisant 23 couches a été entrepris (Rivera, Ledoux, 1991) (fig. 8). Une adaptation du modèle hydrodynamique NEWSAM (Ledoux, Levassor, 1994) a été réalisée pour coupler aux mécanismes d'écoulement les mécanismes de compaction dans les argiles. Dans cette approche, le couplage hydromécanique, phénomène fortement non linéaire, est essentiellement modélisé dans les couches argileuses par le biais d'un coefficient d'emmagasinement spécifique variable, dépendant de la contrainte effective s'exerçant sur la matrice argileuse, elle-même liée à la pression interstitielle de l'eau. Le couplage est finalement décrit par deux coefficients caractérisant l'argile (indice de compression et indice de gonflement) qu'il est possible de mesurer au laboratoire par des essais cedométriques sur des échantillons prélevés in situ.

La figure 8 illustre la subsidence calculée et observée au centre de Mexico eu regard des débits prélevés dans l'aquifère profond.

\section{VII 睓 CONCLUSION GÉNÉRALE}

Selon le problème posé et selon les échelles d'espace et de temps avec lesquelles il est nécessaire de l'étudier, la simulation du comportement d'un aquifère profond doit être abordée avec des niveaux de complexité variés.

Les mécanismes purement hydrodynamiques d'écoulement de l'eau par diffusivité sont bien maîtrisés et il existe de nombreux outils informatiques validés par un grand nombre de cas réels. Cependant leur application à des systèmes profonds reste toujours délicate car il n'existe en général que peu de données dans l'espace et dans le temps pour contraindre le modèle. Le travail de modélisation reste en tout état de cause une tâche de spécialiste.

Plusieurs mécanismes couplés sont susceptibles de compliquer l'hydrodynamique et leur prise en compte est rendue actuellement nécessaire par l'évolution des problèmes posés en hydrogéologie appliquée tels que les stockages souterrains. La description du transfert de matière exige une très bonne validation des vitesses d'écoulement calculées par les modèles. L'utilisation des traceurs naturels est une voie de recherche intéressante pour parvenir à cet objectif.

Des modélisations de plus en plus fines deviennent nécessaires pour l'étude de phénomènes à long terme dont l'expérimentation à une échelle proche de la vraie grandeur n'est pas possible. C'est ainsi que les modèles d'aquifères sont progressivement conduits à intégrer des aspects mécaniques pour simuler la stabilité à long terme des structures géologiques.

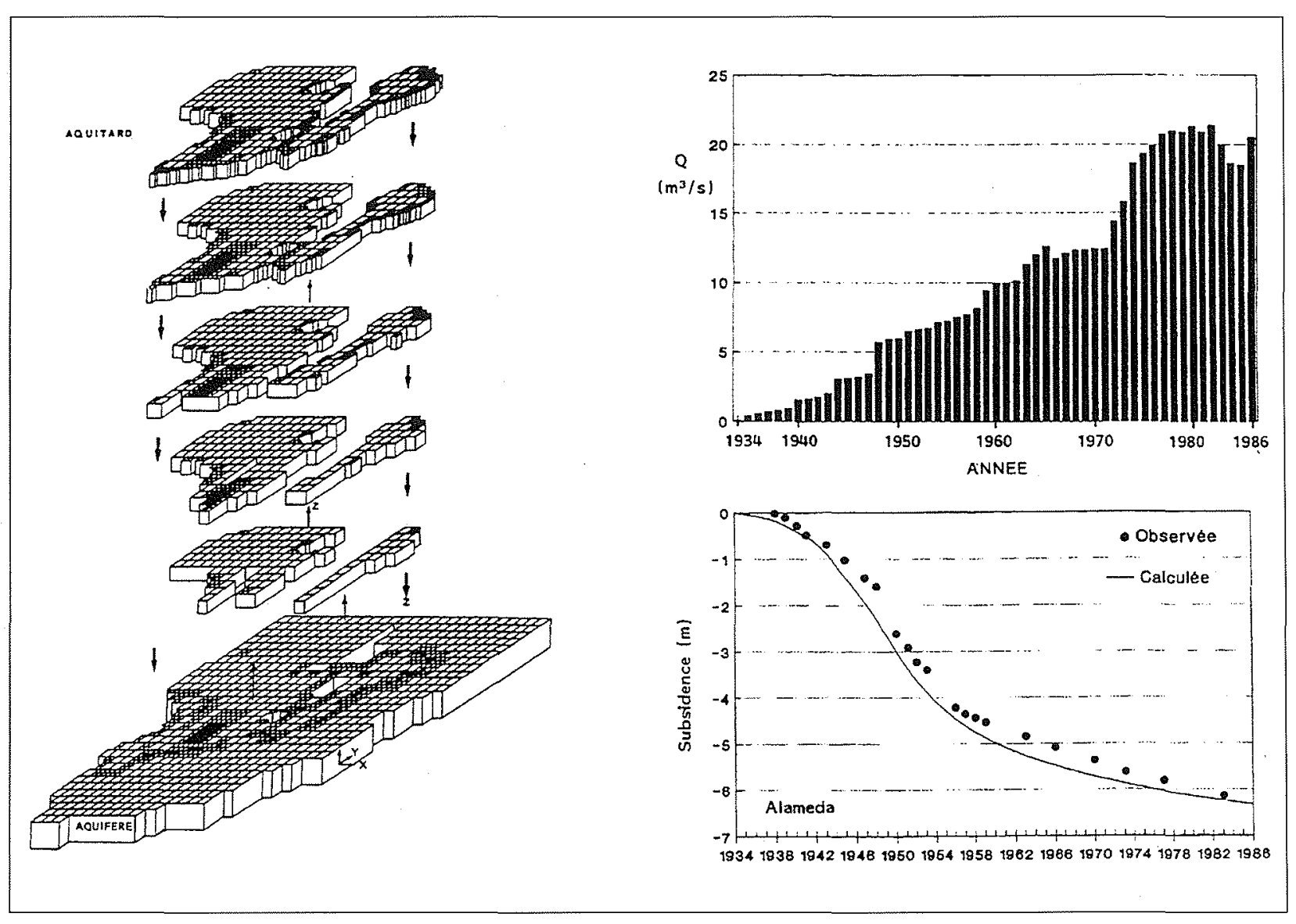

8. Structure du modèle de la nappe de Mexico. Débits pompés entre 1934 et 1986 dans l'aquifère profond et subsidence au centre de la ville (d'après Rivera, 1990). 
D'une manière plus complexe encore, le transport d'éléments réactifs au sein d'un aquifère intéresse un nombre croissant de problèmes appliqués tels la genèse des gisements métalliques ou d'hydrocarbures, ou encore la sûreté des stockages profonds de substances nocives pour l'environnement. Un effort important est actuellement réalisé dans ce but par les chercheurs pour incorporer une phénoménologie géochimique aux modèles d'écoulement et de transport.

La puissance des moyens informatiques communément disponibles permet à présent d'envisager des modèles très complexes couplant des mécanismes hydrauliques, thermiques, mécaniques et chimiques. De tels modèles tridimensionnels sont actuellement en cours d'élaboration pour la simulation des transferts de fluides au sein d'un bassin sédimentaire au cours de son histoire géologique et trouvent leur application dans l'exploration pétrolière.

\section{Références}

[1] Besbes M., Ledoux E., Levassor A., De Marsily G. (1976). - Evolution à long terme de la qualité des eaux souterraines dans un bassin hydrogéologique provoquée par l'activité humaine. Société Hydrotechnique de France, XIV Journées de l'Hydraulique, Paris 1976.

[2] Besbes M., de Marsily G., Plaud M. (1976). - Bilan des eaux souterraines dans le Bassin aquitain. Annales Instituti
Geologi Publici Hungarici, Conférence de Budapest, mai-juin 1976.

[3] Emsellem Y. (1967). - Les transferts de pression entre nappes et la drainance dans les ensembles aquifères hétérogènes. Chronique d'Hydrogéologie $\mathrm{n}^{\circ} 11$, Editions BRGM, pp. 131152.

4] Ledoux E. (1986). - Modèles mathématiques en hydrogéologie. Document Ecole des Mines de Paris, Centre d'Informatique Géologique, LHM/RD/86/12, 118 p.

[5] Ledoux E., Levassor A. (1994). - NEWSAM. Un programme général de simulation des écoulements et des transports de matière dans les systèmes aquifères complexes. NOTICE D'UTILISATION. Document Ecole des Mines de Paris, Centre d'Informatique Géologique, LHM/RD/94/53.

16] De Marsily G., Ledoux E., Levassor A., Potrrinal D., SALEM A. (1978). Modelling of large multilayered aquifer systems, theory and applications, Joumal of Hydrology, 36 (1978), pp. 1-34

[7] Rivera A., Ledoux E. (1991). - Modélisation non linéaire des écoulements et de la compaction dans le système aquifere de Mexico. Société Hydrotechnique de France, XXI Journées de l'Hydraulique, Sophia-Antipolis, 1991.

[8] Wei H.F., Ledoux E., de Marshly G. (1990). - Regional modelling of groundwater flow and salt and envirommental tracer transport in deep aquifers in the Paris basin. Journal of Hydrology, 120 (1990), pp. 341-358. 